\title{
Emergency department overcrowding: Does the Canadian Association of Emergency Physicians have a role?
}

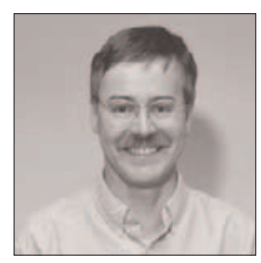

Douglas Sinclair, MD

$\mathrm{T}$ he annual emergency department (ED) overcrowding crisis has begun again, and this year there is tremendous media hype. In fact, over the last few months real emergency physicians have received more attention than the stars of $E R$ !

The Canadian Association of Emergency Physicians (CAEP) Strategic Plan states clearly that CAEP should play an advocacy role and influence public policy as it relates to the provision of emergency care. Recent media attention has given us a window of opportunity to engage both the federal and provincial governments in a dialogue on health care reform. ED overcrowding is a symptom of system failure, and the CAEP Board intends to take a leadership role and do what we can to relieve the pressure now being borne by Canadian emergency departments.

The CAEP Board has a number of initiatives to address this serious problem. To make sensible decisions, we require reliable data on $\mathrm{ED}$ waiting

President, Canadian Association of Emergency Physicians times. The Canadian Emergency Department Triage and Acuity Scale ${ }^{1}$ will help us gather meaningful standardized data, and its widespread detract from our successful education and research initiatives; rather, it reflects the maturity of our organization and speciality.

\section{ED overcrowding is a symptom of system failure, and the CAEP Board intends to take a leadership role and do what we can to relieve the pressure now being borne by Canadian emergency departments.}

adoption is an important development of which we can be proud. In addition, CAEP is actively promoting a national forum at which federal and provincial government representatives can meet ED care providers to discuss problems and develop solutions. We know that many of the solutions lie beyond the sphere of emergency medicine and include primary care reform, improved home care, and increased long term care capacity to ease the bed pressure on acute care institutions. In all of these areas, CAEP will strive to be a leader. At the same time, our expanded advocacy role will not
The CAEP Board (board@caep.ca) welcomes your feedback as we explore these new horizons.

We hope to see many of you in Saint John, NB, for our annual meeting, June 10 to 14, 2000.

\section{Reference}

1. Canadian Association of Emergency Physicians. Canadian Emergency Department Triage and Acuity Scale Implementation Guidelines. CJEM 1999; 1(3 Suppl).

Correspondence to: Dr. Douglas Sinclair, c/o CAEP Head Office, 104-1785 Alta Vista Dr., Ottawa ON K1G 3Y6; board@caep.ca 


\title{
Encombrements des urgences : L'Association canadienne des médecins d'urgence a-t-elle un rôle à jouer?
}

\author{
Douglas Sinclair, MD
}

$\mathrm{V}$ oici de nouveau venu le temps de la crise annuelle d'encombrement des urgences et cette année, celle-ci s'accompagne d'un énorme tapage médiatique. En fait, au cours des derniers mois, les vrais médecins d'urgence ont reçu plus d'attention que les vedettes de $E R$ !

Le plan stratégique de l'Association canadienne des médecins d'urgence (ACMU) indique clairement que l'ACMU devrait assumer un rôle de défenseur d'intérêts et influencer les politiques gouvernementales en ce qui a trait à la dispensation des soins d'urgence. La couverture médiatique récente nous a donné l'occasion d'engager un dialogue sur la réforme de la santé tant avec le gouvernement fédéral qu'avec les gouvernements provinciaux. L'encombrement des urgences est un symptôme de l'échec du système et le Conseil d'administration de l'ACMU entend adopter un rôle de chef de file et faire tout en son pouvoir pour soulager le fardeau qui pèse présentement sur les départements d'urgence canadiens.

Le Conseil d'administration de l'ACMU a établi un certain nombre d'initiatives pour aborder ce problème sérieux. Pour prendre des décisions

Président, l'Association canadienne des médecins d'urgence

intelligentes, il nous faut des données fiables sur les temps d'attente dans les urgences. L'Échelle canadienne de triage et de gravité pour les départements d'urgence ${ }^{1}$ nous aidera à amasser des données normalisées significatives et son adoption répandue

\section{L'encombrement des urgences est un symptôme de l'échec du système et le Conseil d'administration de I'ACMU entend adopter un rôle de chef de file et faire tout en son pouvoir pour soulager le fardeau qui pèse présentement sur les DU.}

représente un progrès important dont nous pouvons être fiers. De plus, l'ACMU fait la promotion active d'un forum national où des représentants des gouvernements fédéral et provinciaux peuvent rencontrer les professionnels de la santé afin de discuter de certains problèmes et trouver des solutions. Nous savons qu'un grand nombre de solutions résident à l'extérieur de la sphère de la médecine d'urgence et qu'elle comprennent la réforme des soins primaires, l'amélioration des soins à domicile et l'augmentation de la capacité des soins à long terme afin de soulager la pression ressentie par les établissements de soins actifs. L'ACMU visera à
Le Conseil d'administration de l'ACMU (board@caep.ca) apprécie vos commentaires alors que nous explorons de nouveaux horizons. Nous espérons vous voir en grand nombre à Saint John (N.-B.), lors de notre assemblée annuelle, du 10 au 14 juin 2000.

\section{Référence}

1. L'Association canadienne des médecins d'urgence: L'échelle canadienne de triage et de gravité pour les départements d'urgence. Guide d'implantation. JCMU 1999;1(3 suppl).

Correspondance : $D^{\mathrm{r}}$ Douglas Sinclair, a/s ACMU, 1785, prom. Alta Vista, Ste. 104, Ottawa ON K1G3Y6; board@caep.ca 